\title{
Numerical Simulation of Aeroacoustic Noise Generated from a Polygon Motor*
}

\author{
Osamu AKIYAMA $^{* *}$, Chisachi KATO ${ }^{* * *}$, \\ Masashi MIYAZAWA $^{* * * *}$ and Takuya YOSHIMURA ${ }^{* * * * *}$ \\ **Konica Minolta Business Technologies, Inc., \\ 2970 Ishikawa-machi, Hachioji-shi, Tokyo, 192-8505 Japan \\ E-mail: osamu.akiyama@konicaminolta.jp \\ ***Institute of Industrial Science, The University of Tokyo, \\ 4-6-1 Komaba, Meguro-ku, Tokyo, 153-8505 Japan \\ ****3-24-3 Honda, Kokubunji-shi, Tokyo, 185-0011 Japan \\ ***** Tokyo Metropolitan University, \\ 1-1 Minami-Osawa, Hachioji-shi, Tokyo, 192-0397 Japan
}

\begin{abstract}
A polygon motor consists of several mirror faces and it is one of the core components for laser beam printers and copiers. Under high-speed rotation, aeroacoustic noise with a peak frequency of rotational speed times the number of mirror faces and its harmonics is prominently generated from a polygon motor covered with a casing. This paper describes a method to predict such noise that is generated from the flow, between the rotating mirror and stationary casing that propagates through the casing and is radiated into the ambient air. The unsteady flow is first computed by large-eddy simulation. The computed pressure fluctuations on the inner wall of the casing are then fed to structural analysis based on a dynamic explicit finite element method that computes the propagation of the elastic waves in the casing. Resulting external surface velocities are finally used for the computation of the acoustical field. A good correlation between the computed and measured results has been observed both in terms of velocity spectra on the casing and the spatial distribution of sound pressure level. The proposed method thus seems a promising engineering tool for predicting and identifying the generation mechanism of such noise that is generated from unsteady flow that propagates through solid walls and is radiated to ambient air, in general.
\end{abstract}

Key words: Structual Noise, Polygon Motor, Aeroacoustic, Large Eddy Simulation

\section{Introduction}

A polygon motor is one of the core component for laser beam printer and a Multifunction Peripheral as shown in figure 1. Rotating polygon motor consists of several mirror faces reflects laser-beams and they plot latent images on a photo conductor drum. A polygon motor generates peak noises at frequency of $n z$ ( $n$ : rotational frequency, $z$ : number of mirror faces) and its harmonics similarly to turbo machinery noise at the blade passing frequency. For high-speed operation of a polygon motor, reduction in the peak noise at high frequency is demanded although the noise level is not necessarily high when it is covered with a casing. This is because such a peak noise has, in general, a high frequency such as ten thousand hertz and is very uncomfortable. As for studying the aeroacoustic noise generated from a polygon motor, flow structures around a low-speed rotating polygon motor in open air are clarified with flow visualization experiments using PIV by Honda and

*Received 10 Feb., 2009 (No. T1-06-0467) Japanese Original : Trans. Jpn. Soc. Mech. Eng., Vol.73, No.725, B (2007), pp.67-75 (Received 27 Apr., 2006) [DOI: 10.1299/jee.4.162] 


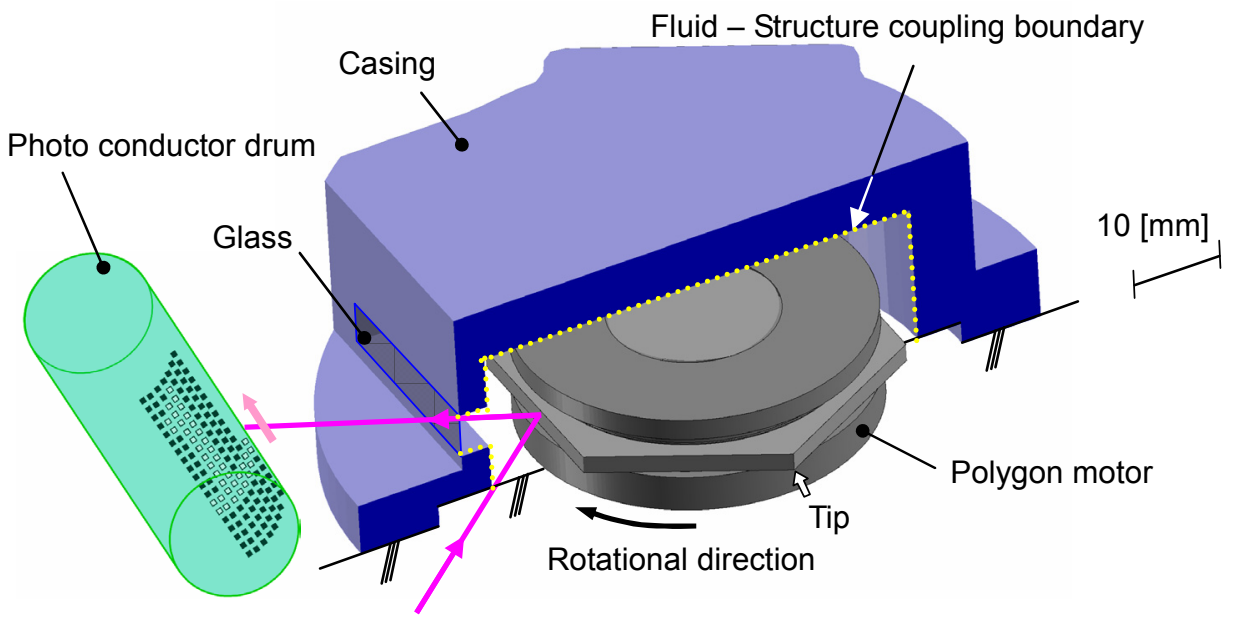

Fig. 1 Polygon motor

Obara[1,2]. However, noise mechanisms such as spatially localized noise sources and propagation paths in a polygon motor with a casing have not been reported as of today.

The mechanism of noise generation is roughly described as follows: 1) rotating polygon mirror faces generate pressure fluctuation on the internal surface of the casing, 2) elastic waves excited by the vibration source propagate thorough the solid casing, and 3) vibration velocity on the external surface of the casing radiate sound waves into the ambient air. Regarding the flow induced noise inside a structure like this case, a prediction method based on a one-way coupling analysis of fluid flow, structural and acoustical computation has been applied to a 5 -stage centrifugal pump[3,4,5,6].

In this study, pressure fluctuations are computed by large-eddy simulation (LES), frequency transfer characteristics and operational deflection shapes radiating noise are then computed by dynamic explicit structural analysis, and finally radiated sound fields are predicted by acoustical analysis. In regards to the noise source at $n z$ and $2 n z$ component, prediction accuracy of the present method is verified by comparisons with measurements in terms of the vibration velocity as well as the radiated sound pressure level (SPL). In addition, sound generating mechanism and propagation characteristic are clarified by the computed results. Although this method neglects acoustic modes in the space covered with a casing, there is no impact as will be shown later.

\section{Inner Flow-Field Computation using LES}

Pressure fluctuations in the casing are computed by Front Flow - Blue, which is an LES code developed by Kato et al $[7,8]$. In this study, we assume that the vibrating aluminum casing excited by pressure fluctuations do not induce flows.

\subsection{Governing Equations}

The governing equations of LES are the spatially filtered continuity equation (1) and the incompressible filtered Navier-Stokes equations (2).

$$
\begin{aligned}
& \frac{\partial \bar{u}_{i}}{\partial x_{i}}=0 \\
& \frac{\partial}{\partial t} \bar{u}_{i}+\frac{\partial}{\partial x_{j}} \bar{u}_{i} \bar{u}_{j}=-\frac{1}{\rho} \frac{\partial \bar{p}}{\partial x_{i}}+\frac{\partial}{\partial x_{j}}\left\{\left(v+v_{S G S}\right)\left(\frac{\partial \bar{u}_{i}}{\partial x_{j}}+\frac{\partial \bar{u}_{j}}{\partial x_{i}}\right)\right\}+f_{i}
\end{aligned}
$$

where

$$
v_{S G S}=\left(C_{s} f \Delta\right)^{2}\left(2 \overline{S_{i j}} \overline{S_{i j}}\right)^{0.5}
$$




$$
\begin{aligned}
& \overline{S_{i j}}=\frac{1}{2}\left(\frac{\partial \bar{u}_{i}}{\partial x_{i}}+\frac{\partial \bar{u}_{j}}{\partial x_{j}}\right) \\
& f=1-\exp \left(-\frac{y^{+}}{A^{+}}\right) ; \quad A^{+}=25.0
\end{aligned}
$$

where, $\overline{u_{i}}$ is the grid-scale velocity component in the $x_{i}$-direction, $\bar{p}$ is the grid-scale static pressure, $\rho$ is the density and $v$ is the kinematic viscosity. $f_{i}$ is the inertial force associated with the motion of the frames of reference. For a rotational frame of reference, the centrifugal forces and Coriolis forces must be added. The effect of eddies that are not resolved by the grid (sub-grid scale eddies) are modeled by Standard Smagorinsky model, and incorporated with the Van-Driest wall-damping function that represents the near-wall effects. The model coefficient $C_{s}$ is fixed to 0.20 , which is a standard value for flows with large separation expected around the downstream of the tips.

\subsection{Numerical Method and Computational Condition}

Using a streamline upwind finite element formulation of second order both in time and space, LES code numerically computes flow fields in this study. A detailed description is given by Kato et al $[9,10]$.

The computed polygon motor model sealed with casing and glass is shown in figure 1 . It is composed of 6 mirror faces with a tip diameter $D_{t}=42.7 \mathrm{~mm}$ that rotates at 63,000 $\min ^{-1}$, resulting in a tip speed of $U_{t}=141 \mathrm{~m} / \mathrm{s}$. The Reynolds number based on this tip speed $\mathrm{U}_{\mathrm{t}}$ and the representative diameter $\mathrm{D}_{\mathrm{t}}$ is $R e=3.98 \times 10^{5}$. There are regions in which the Mach number in the downstream of the tips is 0.3 or over as shown in figure 3 . However, the effect of compressibility is presumably ignorable, such a high Mach number region is localized with a very small area. The computational region that is composed of a combination of rotational and stationary frames of reference is depicted with the computational mesh as shown in figure 2 (a). Each mesh includes appropriate margins of overlap with its neighboring mesh upstream and downstream. At every time step, the velocity components and static pressure within such a margin are the values interpolated in the computational mesh of the corresponding neighbor. The number of grid points is approximately 1 million for a mirror face and 5 million in total, and O-topology grids are adopted for the model. Averaged values of the dimensionless wall distance near the mirrors, in the parallel to flow direction: $x_{\mathrm{m}}$, in the normal to the mirror face direction: $y_{\mathrm{m}}$ and the z-axis direction: $z_{\mathrm{m}}$, are $x_{\mathrm{m}}{ }^{+}=11.9, y_{\mathrm{m}}{ }^{+}=12.4$ and $z_{\mathrm{m}}{ }^{+}=11.4$, respectively. The present

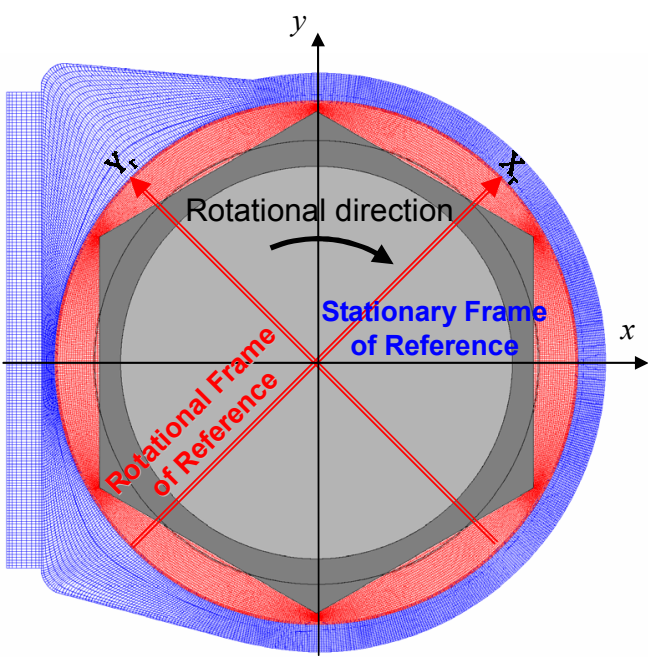

(a) Schematic view of overset grid

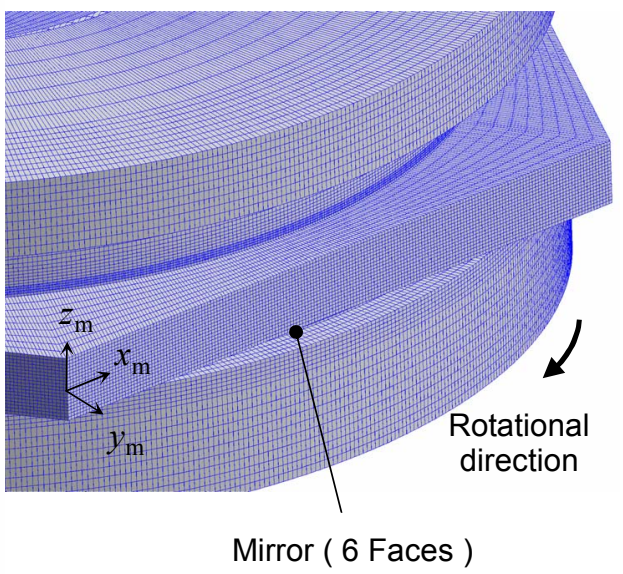

(b) Detail of computational mesh on the rotor

Fig. 2 Computational mesh for the polygon motor 
computation with these mesh resolutions properly captures the voriticity behavior separated from the tips. The wall distance $y_{\mathrm{m}}{ }^{+}$can be calculated by equation (6), using the averaged LES results during one revolution.

$$
y_{\mathrm{m}}{ }^{+}=\frac{u_{\tau}}{v} y_{\mathrm{m}} ; \quad u_{\tau}=\sqrt{v\left(\frac{\partial u_{\mathrm{m}}}{\partial y_{\mathrm{m}}}\right)} y_{\mathrm{m}}=0
$$

here, the velocity gradients on the mirrors $\left(y_{\mathrm{m}}=0\right)$ are calculated by the backward difference method, with the velocity component parallel to the mirror faces at the nearest node.

The closed computational region does not have an inlet/outlet boundary condition, which can be defined as a reference pressure, in general. Although absolute values of the pressure are not calculated, the present method safely filters DC trends, using spatially averaged data in the entire computational domain as reference pressure. In the case of the flow of an incompressible fluid, this process could be carried out without problems in terms of computation, because pressure fields are determined only by instantaneous flow fields. On the solid walls, a no-slip boundary condition was prescribed with the Van-Driest damping function for the Standard Smagorinsky Model. The time increment of the computation was set such that 4,000 time steps corresponded to one revolution of the polygon motor : $\Delta \mathrm{t}=2.38 \times 10^{-7}\left[\mathrm{~s}\right.$ ] (Dimensionless time step based on $\mathrm{U}_{\mathrm{t}}=141 \mathrm{~m} / \mathrm{s}$ and $\mathrm{D}_{\mathrm{t}}=42.7 \mathrm{~mm}$ is $\left.\Delta \mathrm{t}=7.85 \times 10^{-4}[-]\right)$. Starting from an initial flow field in which all of the velocity components and the static pressure were set to zero, the inner flow field became statistically stable within about fifteen revolutions of the polygon motor and remained in a statistical state of equilibrium after that. The computations were successfully carried out until twenty seven revolutions $\left(\mathrm{t}=2.52 \times 10^{-2}[\mathrm{~s}]\right)$.

\subsection{Numerical Results}

Figure 3 shows the computed instantaneous distributions of tangential velocity. This confirms that flow velocity in downstream of a tip is locally fast $\left(u_{\theta} / U_{t}=1.53\right)$ due to the recirculating flow downstream of the tip. The detailed view near a tip is shown in figure 3 with Mach number contours. The Mach number in the reverse flow region is $M=0.4$ or above and reaches a local maximum value of $M=0.64$. However, the effect of compressibility is presumably ignorable because such a high Mach number region is localized with a small area and the entire computational domain is also comparatively small.

Figure 4 shows the computed instantaneous streamlines on $\theta=$ (a) $10 \mathrm{deg}$, (b) $28 \mathrm{deg}$ and (c) $57 \mathrm{deg}$ planes. Streamlines are plotted together with tangential direction component vorticity $\omega_{\theta}$. In the (a) $\theta=10$ degree plane, flows separated at the edge of the upper and

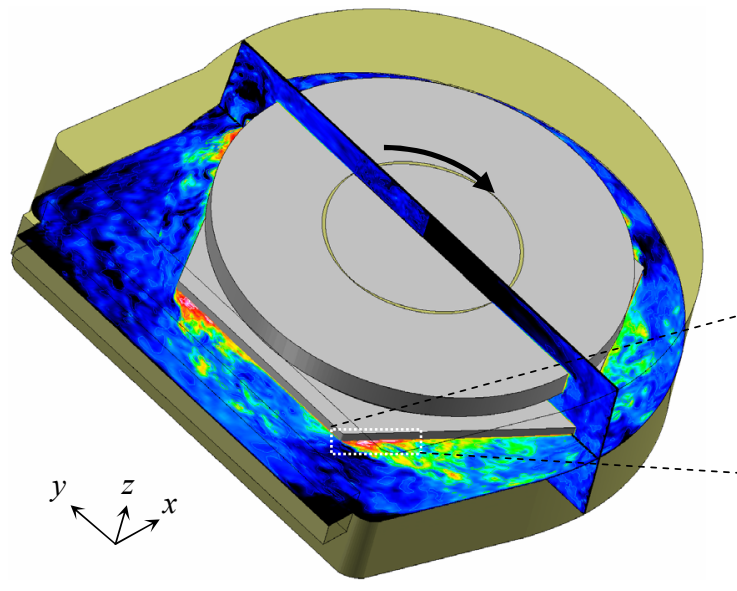

$$
\begin{array}{l|l}
u_{\theta} / \mathrm{U}_{\mathrm{t}} \\
\hline & 1.5
\end{array}
$$

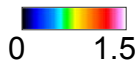

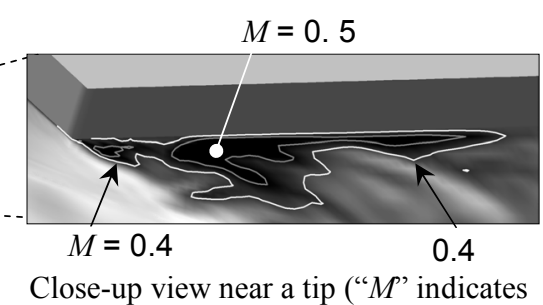

Close-up view near a tip (“ $M$ " indicates local Mach number)

Fig. 3 Computed instantaneous distributions of tangential velocity in the polygon motor's mid-height and $x=0$ planes 


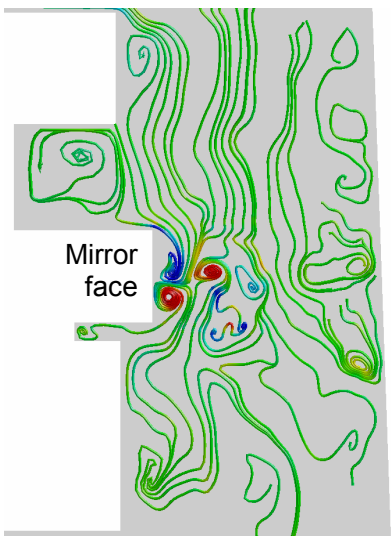

(a) $\theta=10 \mathrm{deg}$

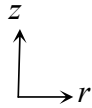

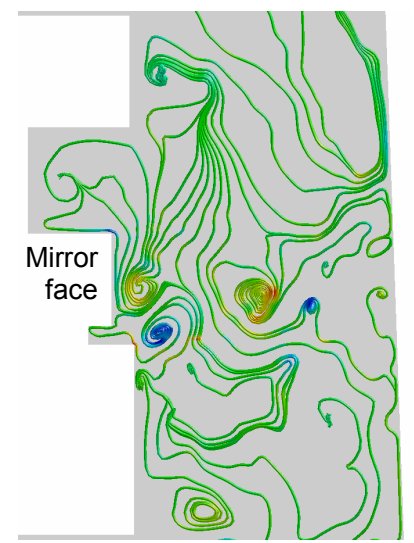

(b) $\theta=28 \mathrm{deg}$

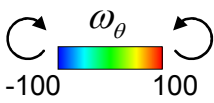

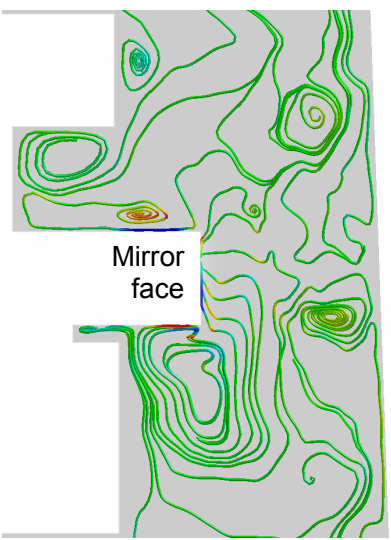

(c) $\theta=57 \mathrm{deg}$

(c) $57 \mathrm{deg}$

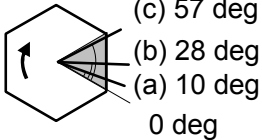

Fig. 4 Computed instantaneous stream line on $\theta=10,28,57$ deg planes colored by vorticity $\omega_{\theta}$
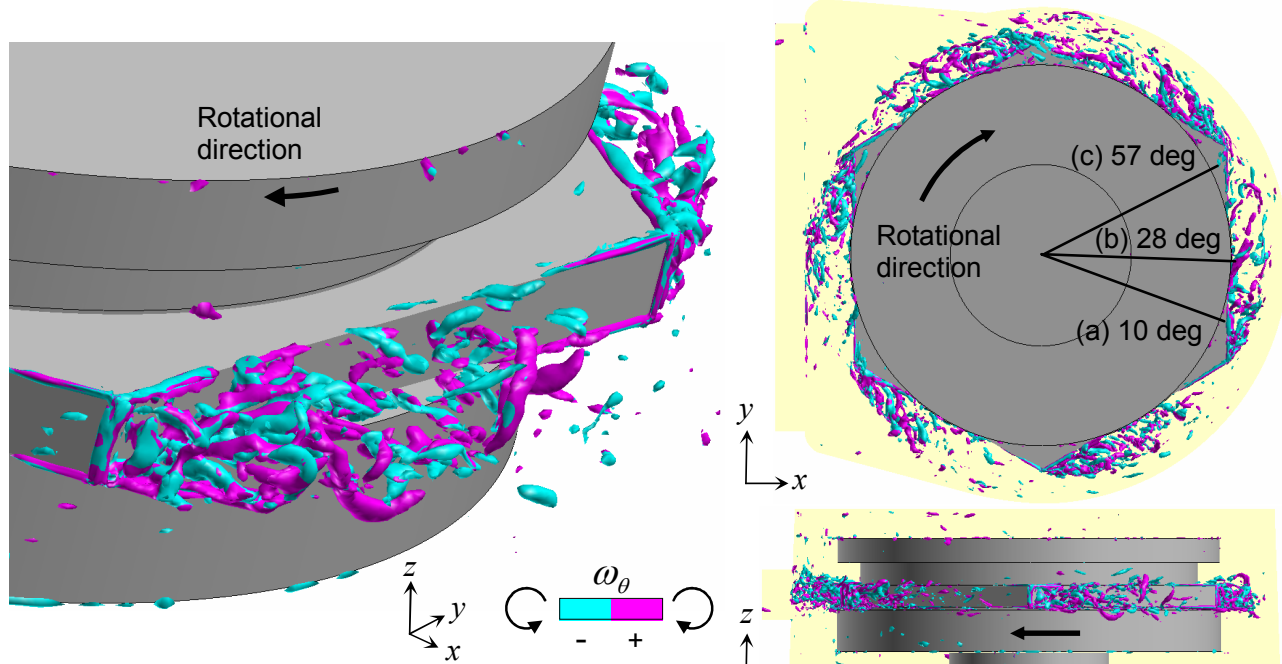

(a) $10 \mathrm{deg}$
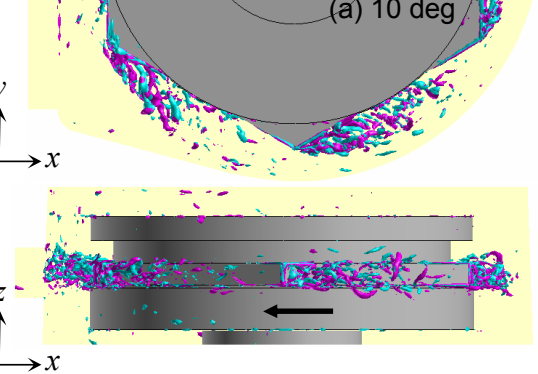

Fig. 5 Computed instantaneous vortical structures around the mirror $\left(\nabla^{2} \mathrm{p}=1000\right)$

lower surfaces come into the above-mentioned recirculating zone and form intense vortices there. In the (b) $\theta=28$ degree plane, some vortices are still generated, but most of the vorticities generated upstream have been and are being destroyed without spreading to radial direction. In the (c) $\theta=57$ degree plane, all the intense vortices have been destroyed. It can be said that vortical motion has no apparent effects on the downstream mirror.

Figure 5 shows the computed instantaneous vortical structures around the mirror with iso-surfaces of $\nabla^{2} \mathrm{p}=1000$. These $\theta=$ constant lines (a)(b)(c) shown in the upper right of figure 5 which corresponds to the planes plotted with streamlines in figure 4 . The static pressure is normalized by twice the dynamic pressure that corresponds to the tip speed $U_{t}$. As already mentioned, three-dimension vortical structures generated behind a tip are destroyed without spreading to the axial and radial directions. The turbulent vortical motion thus has a limited effect behind a tip and no effect on other places such as inner casing or downstream mirror faces. 


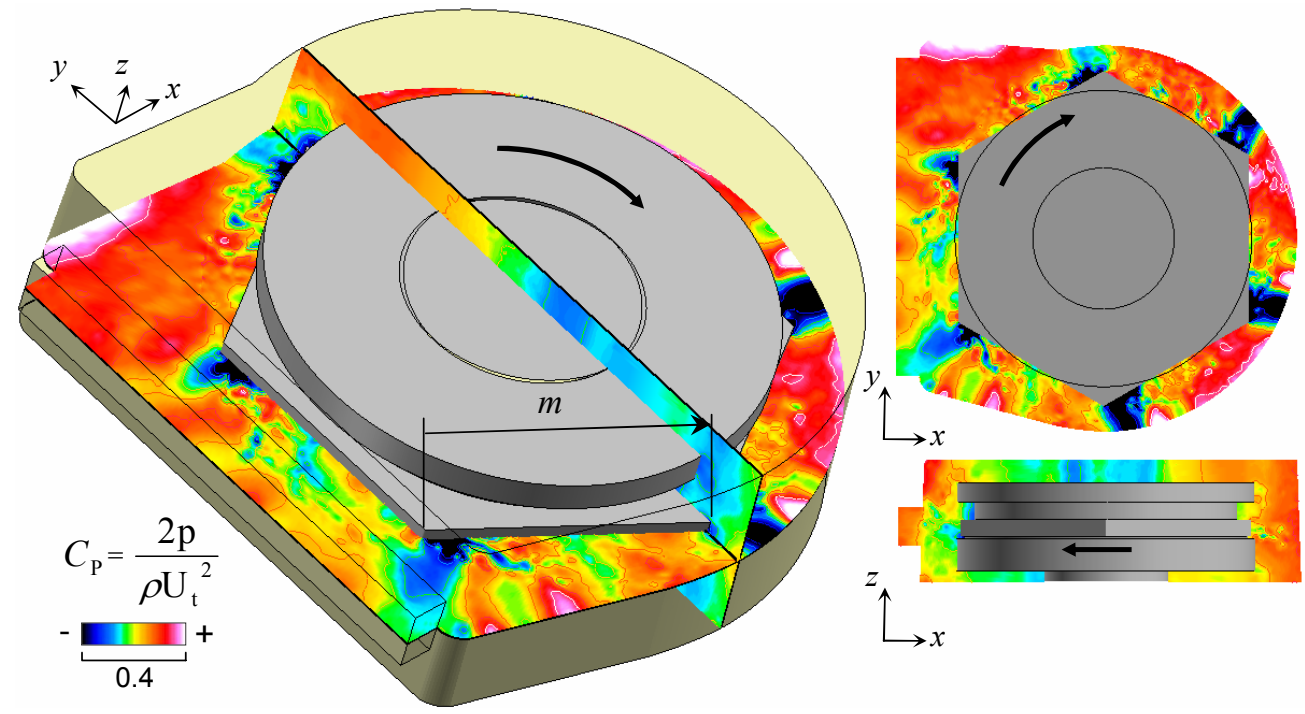

Fig. 6 Computed instantaneous pressure distribution in the mid-height and $x, y=$ constant planes
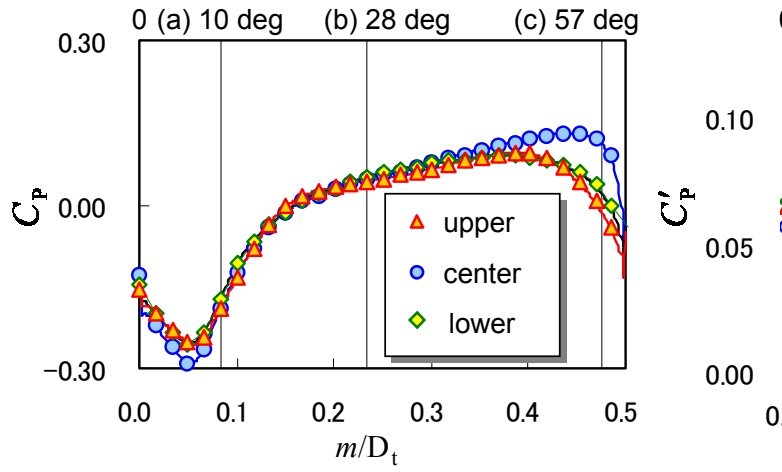

0 (a) 10 deg

(b) $28 \mathrm{deg}$

(c) $57 \mathrm{deg}$

Fig. 7 Computed time-averaged (left ) and fluctuating (right) pressure on a mirror face

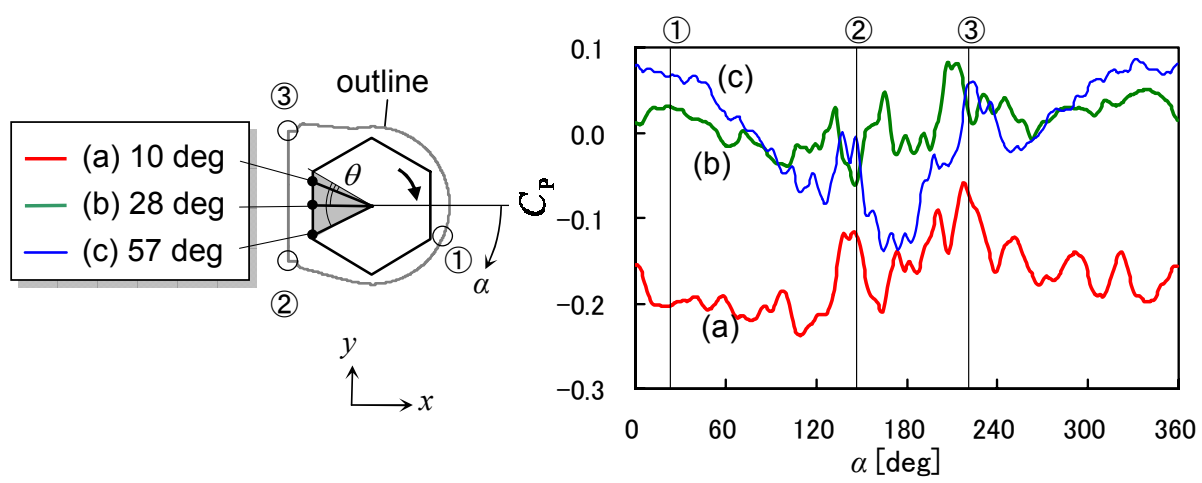

Fig. 8 Computed ensemble averaged pressure at $\theta=10,28,57$ deg point of the mirror face

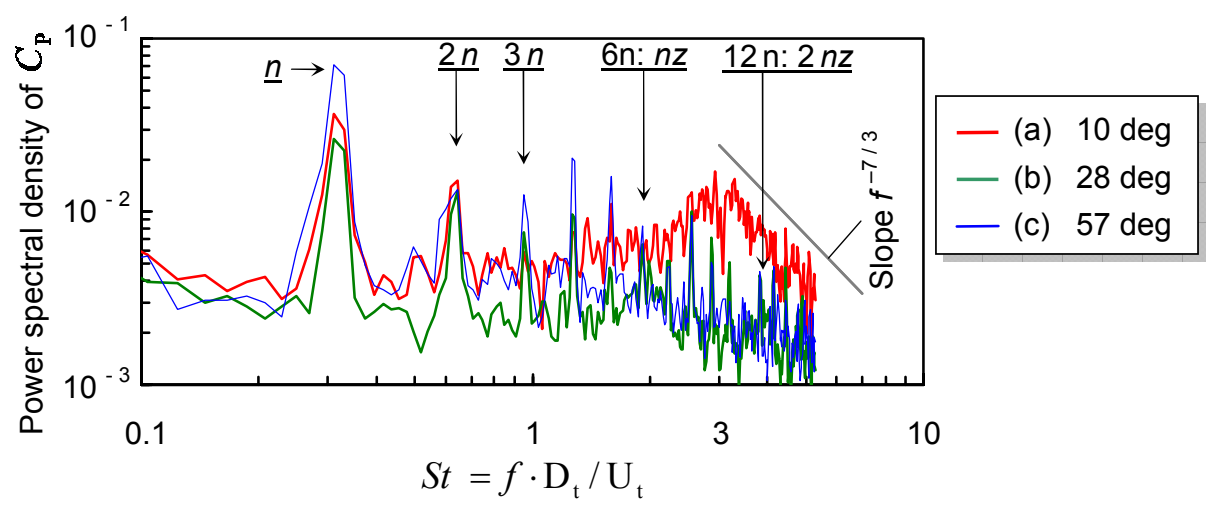

Fig. 9 Computed power spectra of pressure fluctuation at $\theta=10,28,57 \mathrm{deg}$ points of the mirror face 
Figure 6 shows the computed instantaneous pressure distributions. Negative pressure regions (relatively low level of pressure region) lie behind a tip, expand to the radial direction and reach the inner surface of the casing. When it is viewed from the stationary frame, the pressure difference between the front and rear side of a tip generate fluctuating component at $n z$ frequency on the inner surface of the casing.

To focus on the mirror face, better understanding of the pressure field can be obtained by viewing it from the rotating frame. Figure 7 depicts the computed time-averaged $C_{\mathrm{P}}$ and fluctuating pressure $C_{\mathrm{P}}^{\prime}$ on a mirror face. These statistical values are obtained by using flow fields on the six mirror faces during the last three rotations. The three lines show the upper, center and lower distributions against the $m$-axis shown in figure 6 . In the time-averaged pressure $C_{\mathrm{P}}$, the negative pressure region behind a tip $\left(m / \mathrm{D}_{\mathrm{t}}<0.15\right)$ corresponds to the reverse flow region ( $M \geqq 0.4$ in figure 6 ). In the fluctuating pressure $C_{\mathrm{P}}^{\prime}$, the maximum point located at the upper and lower position of $m / \mathrm{D}_{\mathrm{t}}=0.09$ is identical to the $\theta=10$ degree place shown in figures 4 and 5 . This indicates that those vortices originating from the separated flows at the upper and lower surfaces cause the intense pressure fluctuation around $m / \mathrm{D}_{\mathrm{t}}=0.09$ shown in figure 7. Ensemble averaged static pressure distributions at (a)(b)(c) on the mirrors are computed, as shown in figure 8. These statistical values are obtained by using flow fields on the six mirror faces during the last sixteen rotations. When the mirror passes by the corners of (2) and (3) on the casing, static pressure is drastically increased by the pressure difference between the front and rear side of a tip and the extruded section on the surface of the casing. This is also the factor of increasing the pressure fluctuations at the corners on the casing (corresponding to Figure 10 (a)). The reason why the maximum is at (1) will be noted later. Figure 9 depicts the computed power spectra of pressure fluctuation at $\theta=10,28$ and 57 degree points of the mirror face. The rotational frequency $n$ and its harmonics have a prominent peak. Note here

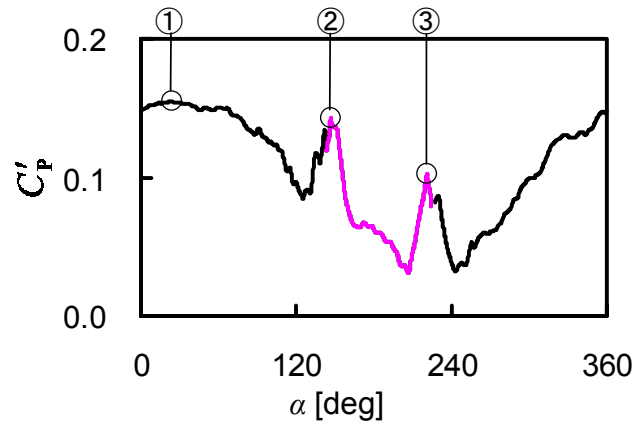

(a) Distribution of pressure fluctuation along the casing ( mid-height )

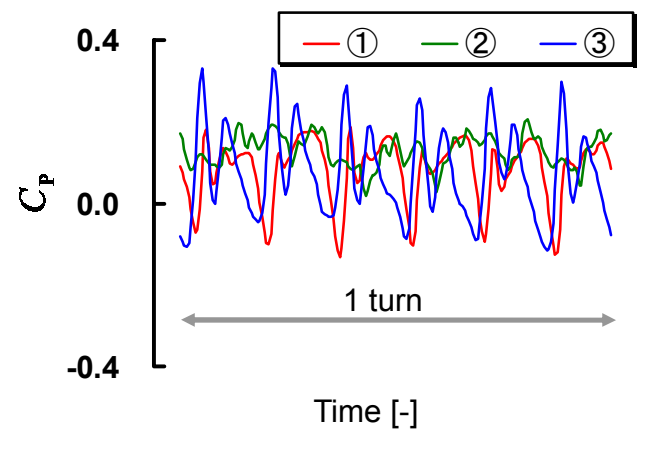

(b) Static pressure history on the casing

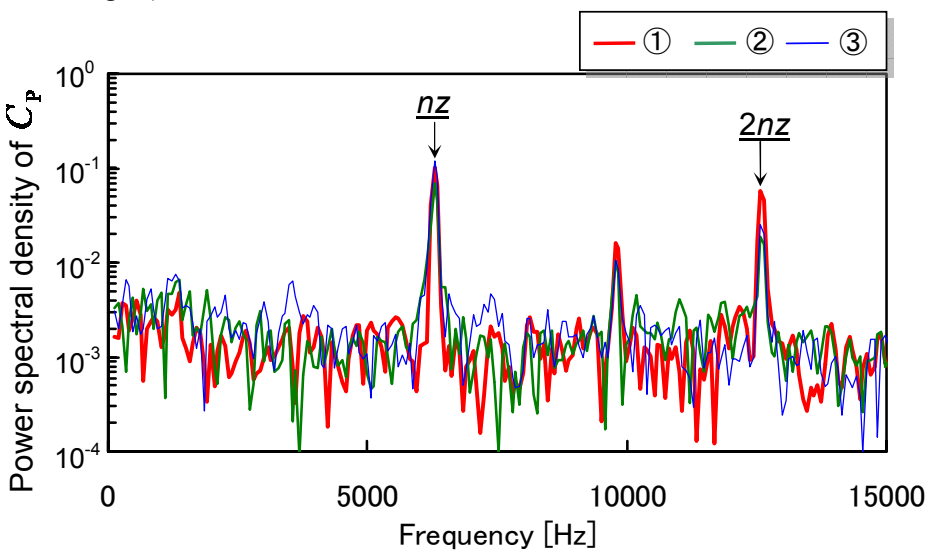

(c) Power spectra of pressure fluctuations on the casing

Fig. 10 Computed static pressure fluctuations on the cashing inner surface 
that $6 n, 12 n$ components are not dominant as compared with other harmonics components. At $\theta=10$ degree point, where vortical motion is most activated as mentioned above, the computed power spectrum of the fluctuating pressure in the range $S t=3-5$ closely obeys what is expected for the inertial subrange of turbulence. Present LES, therefore, captures the vortex structures with appropriate mesh resolution in a target frequency band.

To focus on the casing, which will be set as an exciting boundary condition in the next structural analysis, computed pressure fields on stationary flame have to be discussed more in detail. Computed pressure fluctuation $C_{\mathrm{P}}^{\prime}$ on the inner surface of the casing (z-axis: same as the middle of the mirrors) is shown in figure 10 (a). Pressure fluctuations $C_{\mathrm{P}}^{\prime}$ have a local maximal at (2) and (3) where the corners consist of right angles, confirming the above-mentioned described in figure 8 . Next, we will comment on the periodic component with maximum peak at (1). When it is viewed from the rotational frame, static pressure history at (c) on the mirrors have the periodic component due to the off-centered outline of the casing (see also figure 8). When it is viewed from the stationary frame, distribution of the pressure fluctuations along with the casing also shows a geometrically periodic component shown in figure 10 (a). In other words, to reduce potential sound sources, it is important to avoid the sharp corners and the off-centered outlines of the casing. We believe they are reasonable opinions, because the optimized shape of the two factors has to be a perfect circle. Figure 10 (b) shows the computed static pressure histories per revolution at position (1)-(3) according to Figure 10 (a). The components $n z$ and $2 n z$ corresponding to 6 and 12 peaks are very prominent and strongly auto-correlated. In the next structural analysis, these history data will be fed to the outer surface of the casing as an excitation force boundary condition. Figure 10 (c) plots the computed power spectra of pressure fluctuations at point (1)-(3) in the casing inner surface ( in the stationary reference frame). The frequency characteristic of sound source has a prominent peak at $n z$ and $2 n z$ components. Note here that $n z$ component is greater than $2 n z$ one. The feature is identified not only at these three points, but also at other points.

\section{Elastic Wave Propagation Analysis using Dynamic Explicit Finite Element Method}

Elastic wave propagation in the casing is computed by NEXST- Impact [11], which is a structural analysis code based on a dynamic explicit finite element method. The structural analysis treats computed pressure fluctuations mentioned in the preceding chapter as excitation forces. The computed velocities on the outer surface of the casing are compared with the measured velocities.

\subsection{Explicit Finite Element Method}

Equation of motion for dynamic elastic vibrations of structure is expressed as,

$$
[\mathrm{M}][\ddot{x}\}+[\mathrm{C}]\{\dot{x}\}+[\mathrm{K}]\{x\}=\{f\}
$$

$$
[\mathrm{C}]=\mathrm{c}_{\mathrm{k}}[\mathrm{K}]+\mathrm{c}_{\mathrm{m}}[\mathrm{M}]
$$

where, $\{x\},\{\dot{x}\},\{\ddot{x}\}$ are the displacement, velocity and acceleration vectors, respectively. $[\mathrm{M}],[\mathrm{C}]$ and $[\mathrm{K}]$ are the mass, damping and stiffness matrices, respectively. $\{f\}$ is the load vector. Assuming that Rayleigh damping is applicable to the casing, the damping matrix [C] is expressed as Equation (8). Where, $c_{m}$ is evaluated through an impact test and the corresponding computation, while $c_{k}$ is assumed to be zero. 


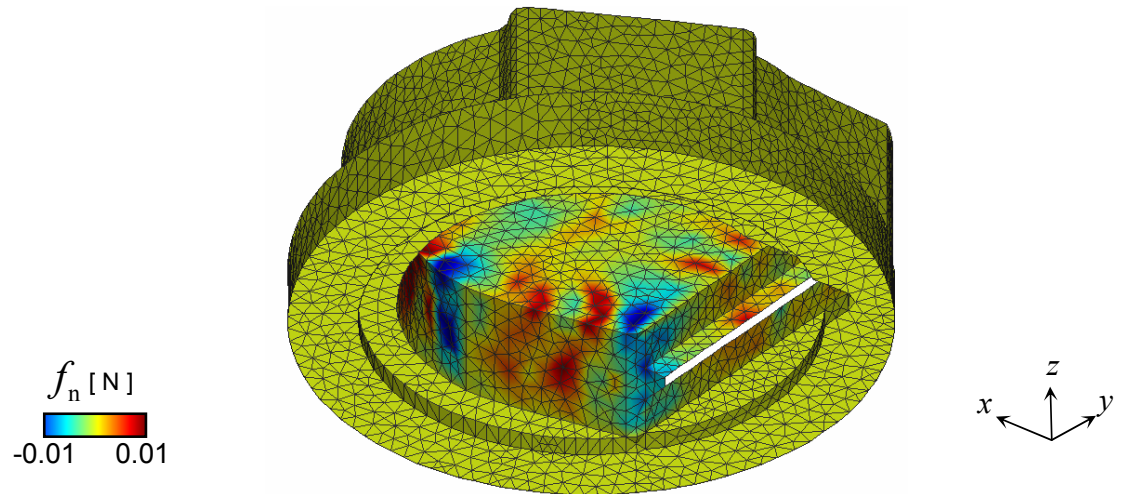

Fig. 11 Computational mesh with instantaneous force boundary conditions mapped for structural analysis
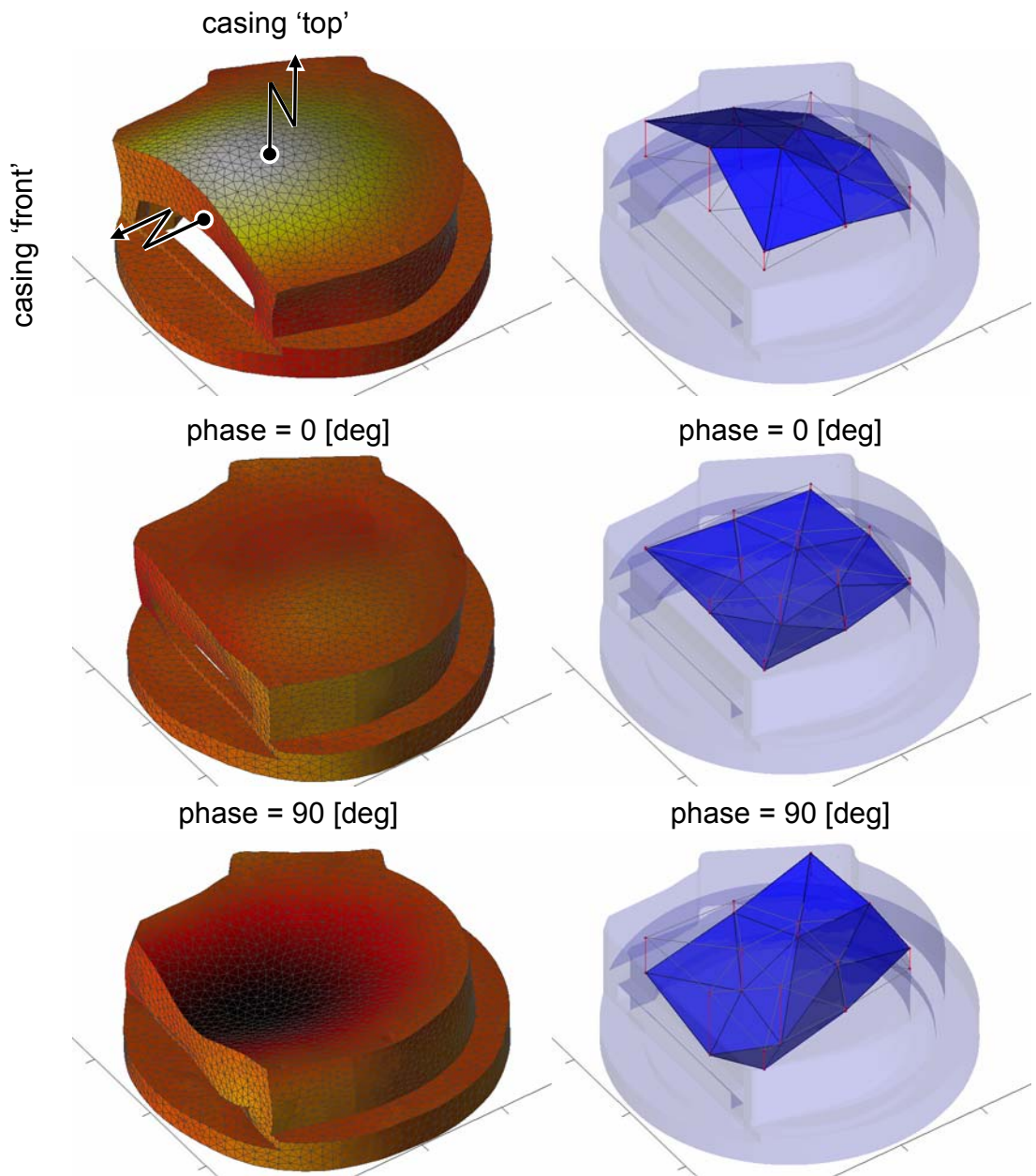

phase $=180[\mathrm{deg}]$
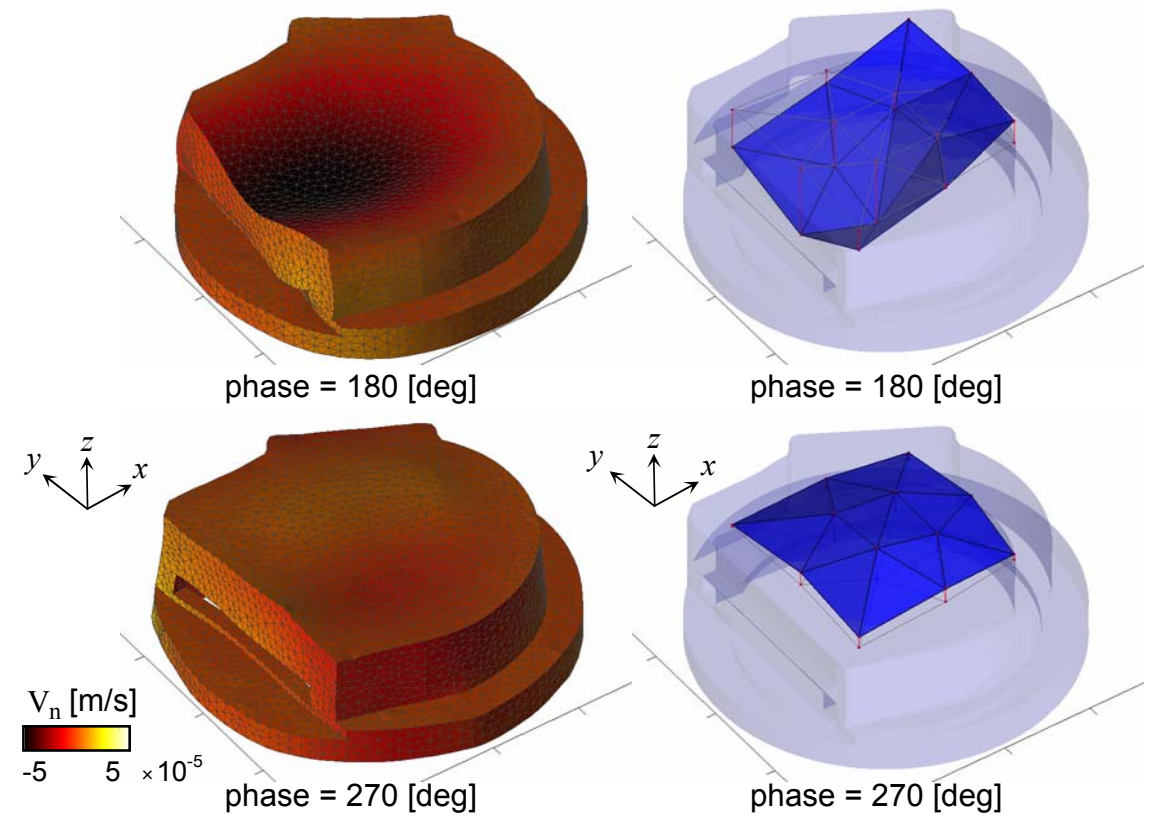

Fig. 12 Distribution of computed normal Fig. 13 Measured deflection shape of the casing velocity at $2 n z(12.6 \mathrm{kHz})$ top at $2 n z(12.6 \mathrm{kHz})$ in operation 


\subsection{Computational Conditions}

Pressure fluctuations computed by the LES feed the load matrix $\{f\}$ in a form of the time history data [5]. The connection and contact boundary conditions for pairing the flange and basement surface are identified such that the computed natural frequency agrees with the measured one. Figure 11 shows the computational mesh for the structural analysis together with the mapped force boundary condition. The aluminum casing model with an outer diameter of $64 \mathrm{~mm}$ and wall thickness of $7 \mathrm{~mm}$ is composed of approximately 50,000 tetrahedral elements, and the glass part for transmitting laser beams is ignored.

\subsection{Numerical Results}

Figure 12 shows the distribution of computed normal velocity $\mathrm{V}_{\mathrm{n}}$ at $2 n z(12.6 \mathrm{kHz})$. The deflection behavior shown in figure 12 is the same as one of the natural modes computed by an eigen-value analysis although it is not shown here. Figure 13 shows a measured deflection shape of the top of casing at $2 n z$ in operation. The measured deflection shape is very similar to the computed one (figure 12). This is the first membrane oscillatory mode that efficiently radiates acoustic noise, in general. The present structural analysis seems to be a method that successfully computes the characteristic deflection shape, which radiates sound waves into the ambient air.

Figure 14 shows a comparison of the normal velocity at the 'top' and 'front' of the casing where Power Spectrum Density (PSD) of the measured and computed velocites are plotted. Both the computed and measured velocity have the same trend in which magnitude of $2 n z$ component is greater than $n z$ one. This trend is apparently the result of the propagating characteristics of the structure at $12.0 \mathrm{kHz}$. Note that the experimental results have several other peaks such as rotational frequency $n(1,050 \mathrm{~Hz})$ and its harmonics components generated due to the imbalance of the rotor weighted, and $8 n(8.4 \mathrm{kHz})$ component excited by the motor coil that are not considered in the present computational model. The peak levels at the front side of the casing, shown in the right figure, are not the principle noise sources, because the peak levels at the top of the casing are greater than the front. The present structural analysis cannot predict the frequency characteristics at the front side of the casing, probably due to the glass ignored. Although the viscous damping is most likely to be dependent on the place as well as on the frequency, it is treated as a single constant $\mathrm{c}_{\mathrm{m}}$ in the present study. A more sophisticated model of the viscous damping will perhaps improve the prediction accuracy in the future.

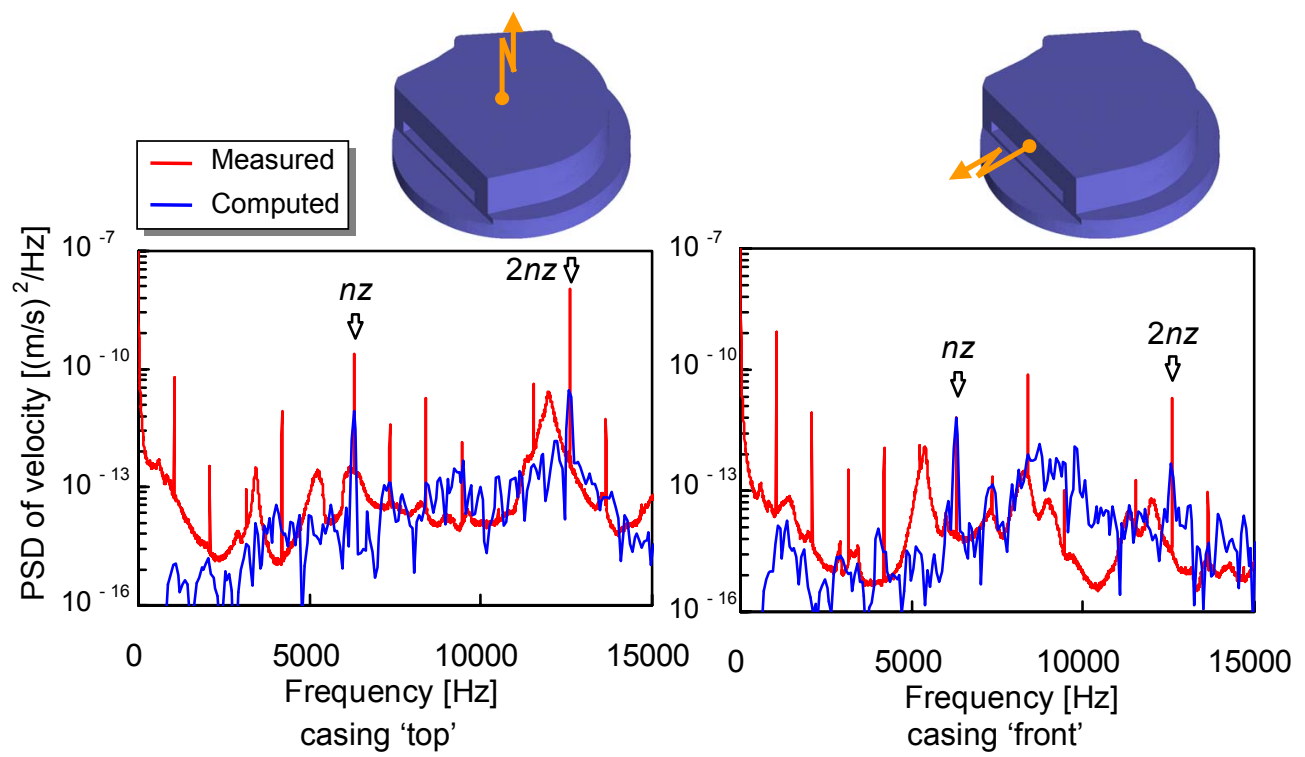

Fig. 14 Comparison of normal velocity at the top and front of casing 


\section{Acoustical Analysis of Sound Field}

Sound fields generated from the casing are predicted in the frequency domain by a commercial acoustical analysis code SYSNOISE $[12,13,14]$. By applying the particle velocity computed on the external surface of the casing by the structural analysis, is computed at arbitrary monitor points in a semi-sphere. The computed sound pressure levels are compared with the measured one.

\subsection{Numerical Method and Computational Conditions}

Sound wave propagations in the ambient air are predicted using a boundary element method for the Helmholtz equations. The computational mesh for the acoustical computation is composed of approximately 5,000 three-node triangle elements with a relatively high resolution where six or more elements are used per wave length of the sound. All reflection condition is given on the $\mathrm{z}=0$ plane in order to model sound radiation in a semi-anechoic room where the measurement was made. The velocity distributions computed by structural analysis (figure 12) are transferred to the acoustical mesh shown in figure 15. SPL is computed on a hemisphere at $r=1.0 \mathrm{~m}$ distance.

\subsection{Numerical Results}

Figure 16 compares the predicted and measured SPL at monitor point $10(r=1.0 \mathrm{~m}$ point in the z-direction). Note here that the predicted SPL has a different frequency resolution from that in the measurement and therefore its level is adjusted to compensate this difference. As already mentioned, the polygon motor noise was measured in a semi-anechoic room, at Tokyo-site, KONICA MINOLTA, with a set of non-directional microphones in accordance with ISO3744 shown in Figure 17. The present computation

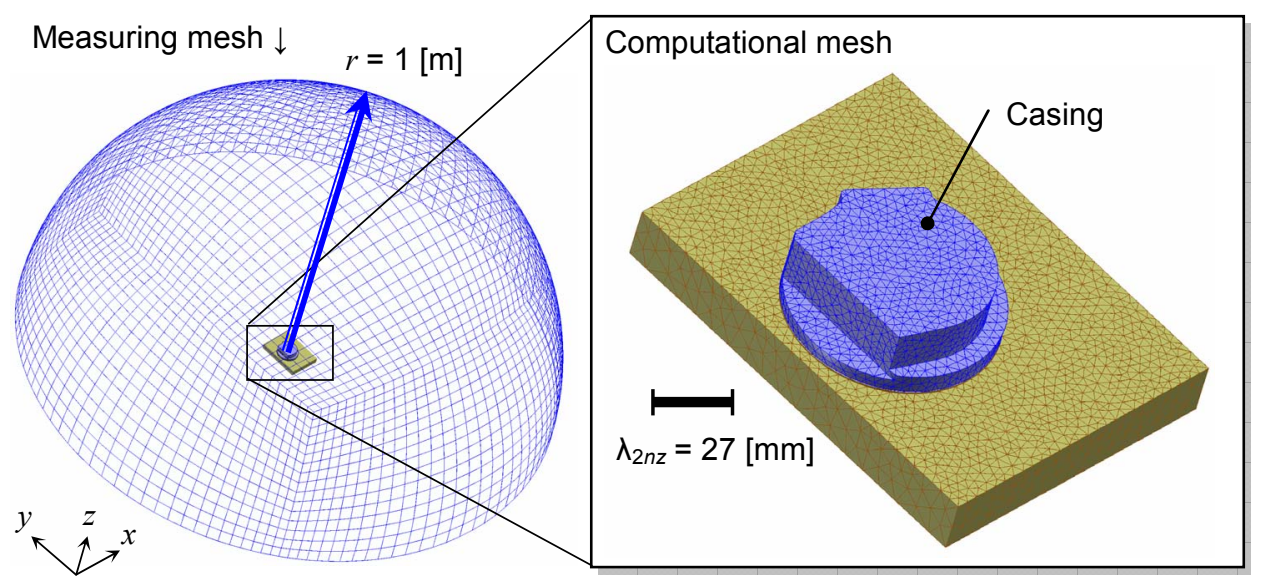

Fig. 15 Computational mesh used for acoustical analysis based on Boundary Element Method

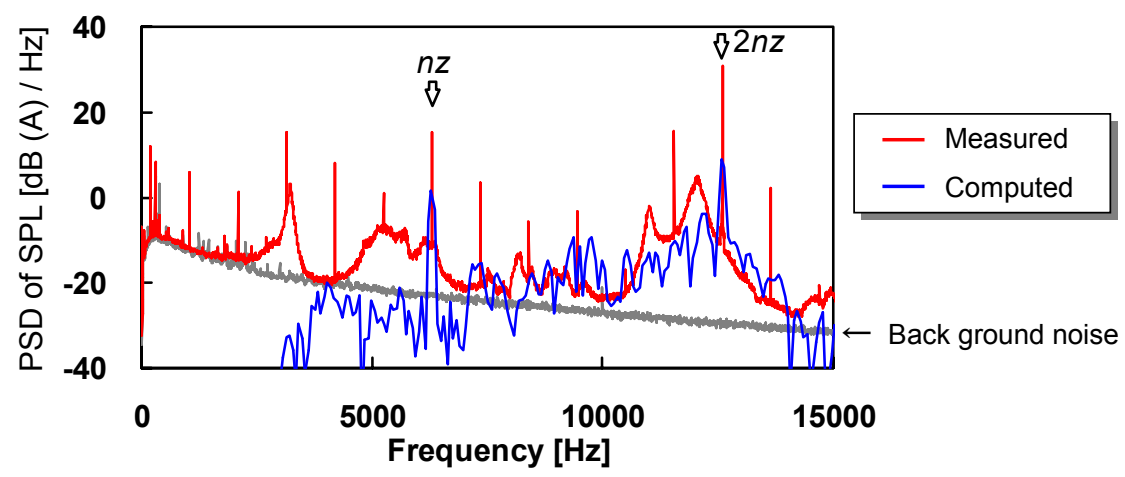

Fig. 16 Comparison of sound pressure level at monitor point 10 ( $z$-axis direction, $1.0 \mathrm{~m}$ ) 
successfully predicts that $2 n z$ component is greater than $n z$ component and dominates the overall noise level, although the computed absolute values of these two peak components are $10-20 \mathrm{~dB}$ lower than the measured equivalents. Regarding the broadband component, the slope around the $2 n z$ peak (at $12.6 \mathrm{kHz}$ ), which is mainly the result of the structural characteristics, are quantitatively predicted by the present computation. As already mentioned, other peak components are produced by what is not modeled in the present study and thus not predicted. Figure 18 shows the computed SPL distributions on the measuring mesh at $2 n z$ component, which is the principle frequency component of the noise. Sound waves strongly propagate only thorough the $z$-axis direction, it is due to the directionality effect at $12.6 \mathrm{kHz}$ ( wave length $\lambda_{2 n z}=27 \mathrm{~mm}$ ). Figure 19 shows comparisons of the SPL at $2 n z$ component at all the monitor points. Note that the measured and computed SPL refer to a different axis, respectively to the left and right axes. Although the computed SPLs are by around $20 \mathrm{~dB}$ lower than the measured ones, the directivity of the sound radiation where intense sound is radiated to the upward direction is reasonably well predicted. The principle mechanism of sound radiation is then the 1 st membrane oscillatory mode of the casing that is excited by the 2nd harmonics of the fluctuating pressure generated by the polygon motor. Therefore, it is very important to predict deflection shapes and natural frequencies on a casing for noise reduction.

Both structural and acoustical analyses quantitatively predict the broadband component while they underpredict the peak components as shown in figure 14 and 16 . The discrepancy is, therefore, attributed to the flow field computation and/or the structural analysis. The structural analysis, however, is more likely to be the cause because it does not predict those peaks at $3.4 \mathrm{kHz}$ and $5.3 \mathrm{kHz}$ that are confirmed by a hammering test.

Finally, we comment on the acoustical mode inside the casing that was ignored in the present analysis. From the inner diameter $48.4 \mathrm{~mm}$ of the casing, the frequency of the fundamental mode is estimated to be around $7.0 \mathrm{kHz}$. However, no strong peaks exist in the

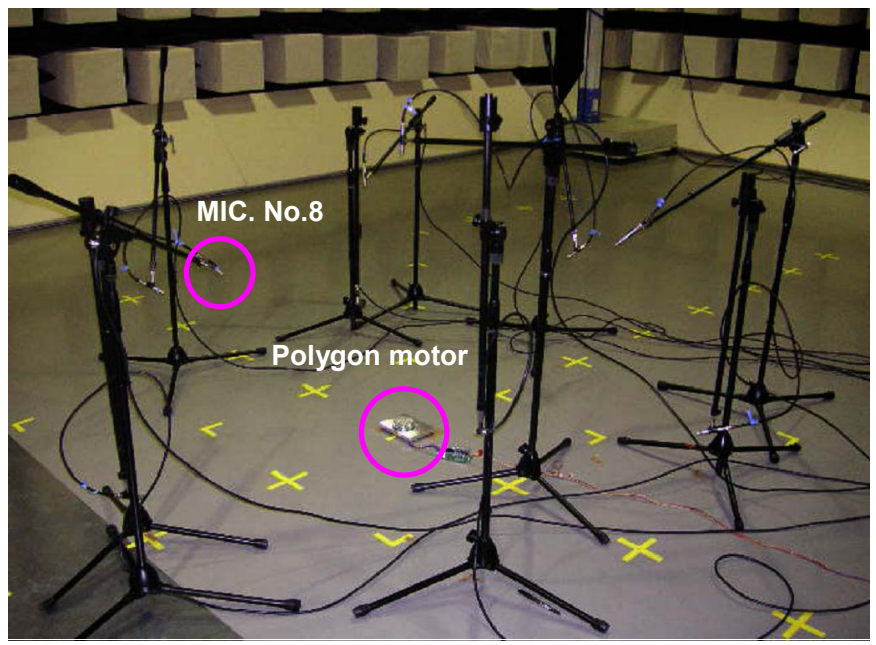

Semi-unechoic room

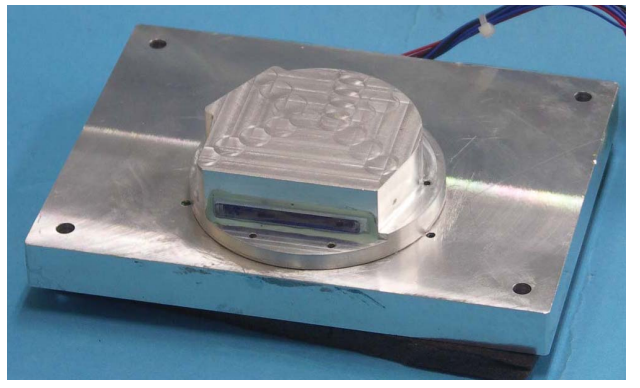

Polygon motor covered with the casing and grass

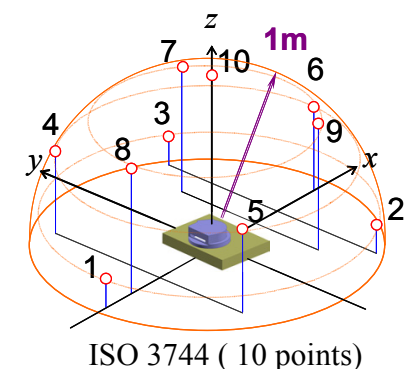

ISO 3744 ( 10 points)

Fig. 17 Photos of the experimental set up 


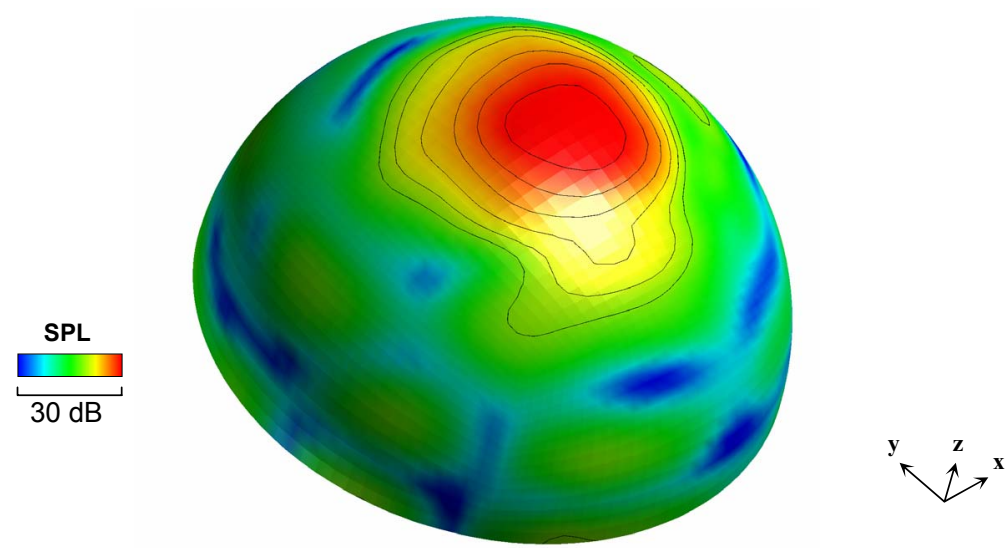

Fig. 18 Computed distribution of sound pressure level on a hemisphere at $r=1.0 \mathrm{~m}$ distance ( $2 n z: 12.6 \mathrm{kHz})$

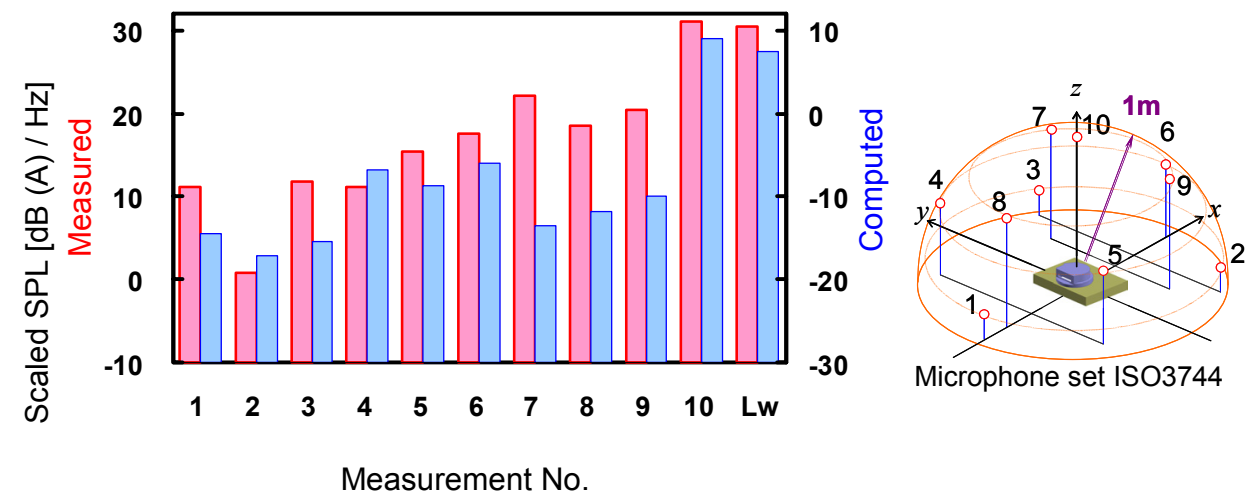

Fig. 19 Comparison of scaled sound pressure level at measurement points $(2 \mathrm{nz}: 12.6 \mathrm{kHz})$

measured vibration velocity or in the measured sound pressure. Therefore, the internal acoustical mode can be safely neglected in this case. Although, this may not be the case for a different number of mirror faces and/or rational speed for which the frequencies of the source fluctuations are different.

\section{Conclusions}

This paper proposes a fluid-structure-acoustic coupling analysis method that is capable of predicting aeroacoustic noise generated from a polygon motor. By comparisons with measurements, the accuracy of the proposed method is verified in terms of the velocity spectra on the casing and the spectrum of SPL of the radiated sound. The method thus seems a promising engineering tool that can be applied to the prediction and reduction of noise generated through a mechanism similar to the one we have presented here.

In this case, the principle mechanism of noise generation is summarized as follows. From the computational fluid analysis, it is clear that the rotating mirror generates pressure fluctuations at $n z$ and $2 n z$ components on the inner surface of the casing. In the structural analysis, the first membrane oscillatory mode at the top of the casing strongly radiates acoustic noise at $2 n z$ component. This is due to the frequency characteristics near the natural frequency at $12.0 \mathrm{kHz}$. In the acoustic analysis, the intense noise is radiated to the $z$ - axis direction at $2 n z$ component, which results in the principle noise.

The following issues should perhaps be considered for possible improvement in the prediction accuracy of the proposed method: 1) a more sophisticated model for viscous damping used in the structural analysis and 2) inclusions of the effects of the acoustical models that may develop inside the casing. 


\section{Acknowledgements}

The authors gratefully acknowledge the benefit of advice and cooperation from Yoshinobu Yamade of Mizuho Information \& Research Institute, Inc. and Dr. Yu Yan Jiang of AdvanceSoft Co., LTD. The computation codes used in this paper, Front Flow-Blue and NEXST-Impact, have been developed by 'Frontier Simulation Software for Industrial Science' project sponsored by the Ministry of Education, Culture, Sports, Science and Technology (MEXT).

\section{References}

(1) Honda, Y. et al., Flow Structure around a Polygon Mirror Inducing Aerodynamic Noise, Proceeding of The Mechanical Engineering Congress 2003, Vol. 4, Tokushima (2003), pp. 313-314 (in Japanese).

(2) Obara, H. et al., Flow Structure around a Rotating Polygon Mirror, The Fifth JSME-KSME Fluids Engineering Conference (2002), 152.

(3) Kato, C. et al., Prediction of the Noise from a Multi-Stage Centrifugal Pump, ASME FED SM2005-77312.

(4) Wang, H. et al., Large Eddy Simulation of Unsteady Flow in a Centrifugal Pump, Proceeding of The 3rd International Symposium on Fluid Machinery and Fluid Engineering, Beijing, China (2004), pp. 168-173.

(5) Jiang, Y.Y. et al., Numerical Simulation of Fluid-Structure coupled Vibration-Flow Induced Noise in a Boiler Feed Pump, Proceedings of The 3rd International Symposium on Fluid Machinery and Fluid Engineering, Beijing, China (2004), pp. 161-167.

(6) Takano, Y. et al., Radiated Noise Simulated from a Full-Scale Multi-Stage Centrifugal Pump, Proceeding of the Twelfth International Congress on Sound and Vibration, Lisbon, Portugal (2005), 731.

(7) Kato, C., Ikegawa, M., Large-Eddy Simulation of Unsteady Turbulent Wake of a Circular Cylinder using the Finite-Element Method, ASME FED, Vol. 117(1991), pp. 49-56.

(8) Kato, C. et al., Large-Eddy Simulation of Unsteady Flow in a Mixed-Flow Pump, International Journal of Rotating Machinery, Vol. 9 (2003), pp. 345-351.

(9) Kato, C. et al., An Overset Finite-Element Large-Eddy Simulation Method with Application to Turbomachinery and Aeroacoustic, Transactions of the ASME, Journal of Applied Mechanics, Vol. 70 (2003), pp. 32-43.

(10) Kato, C., Numerical Prediction of Sound Generated from Flows with a Low Mach Number, Transactions of the Japan Society of Mechanical Engineers, Series B , Vol. 71, No. 711 (2005), pp. 2613-2621 (in Japanese).

(11) Oishi, A. et al., A parallel Explicit Finite Element Analysis based on the Hierarchical Domain Decomposition, Transactions of the Japan Society for Computational Engineering and Science, Vol. 3 (2001), pp. 131-136 (in Japanese).

(12) von Estorff, O. et al., Sound Radiation of a Cantilever Plate Comparisons of BEM Results with Experimental Data, Betech 91, 6th International Conference on Boundary Element Technology, Southampton, U.K.(1991), pp. 147-158.

(13) Coyette, J. P., A Refined Boundary Element Technique for Modeling Acoustic Radiation from Complex Structural Models, 17th International Seminar on Modal Analysis, (1992).

(14) Coyette, J. P., Wynendaele, H., Synthesis of available Boundary Element Formulations for solving Acoustic and Elasto-acoustic Problems, 11th International Modal Analysis Conference, (1992). 